\title{
Ouvrages renforcés : approche par superposition de milieux continus et traitement numérique
}

D. GARNIER

B. SUDRET

ENPC-CERMMO

6-8, av. Blaise-Pascal

Cité Descartes

Champs-sur-Marne

77455 Marne-la-Vallée

Cedex

garnier@cermmo.enpc.fr

\section{E. BOURGEOIS}

J.-F. SEMBLAT

LCPC

58, bd Lefebvre

75732 Paris Cedex 15

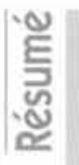

L'analyse par des méthodes numériques du comportement des ouvrages renforcés par inclusions est difficile et coùteuse lorsque I'on veut modéliser explicitement les renforcements. Une approche alternative consiste à remplacer le massif renforcé par un matériau équivalent constitué par la superposition de plusieurs milieux continus, Contrairement aux théories d'homogénéisation "classiques $\%$, cette méthode permet la prise en compte de cinématiques différentes pour chacun des milieux qui constituent le matériau équivalent, et donc de représenter le glissement relatif du sol et des inclusions de renforcement. Elle est en outre plus simple et plus rapide à mettre en œuvre. L'intrégration de ce modèle dans le progiciel CESARLCPC a été entreprise. La version actuelle du code permet le calcul élastoplastique d'ouvrages bidimensionnels et tridimensionnels, dans le cas où seuls les efforts de traction-compression dans les inclusions sont pris en compte, et où la cinématique de chaque matériau constituant est supposée identique. On présente ici les résultats obtenus pour l'étude de deux ouvrages renforcés (analyse tridimensionnelle du tassement d'une fondation carrée reposant sur des pieux, étude de la convergence d'un tunnel boulonné), mettant en évidence la pertinence et lefficacité de la méthode.
NDLE: Les discussions sur cet article sont acceptées jusqu'au $1^{\text {ot }}$ septembre 2003.
The analysis by numerical methods of the behavior of the reinforced works by means of inclusions is difficult and expensive when the reinforcements are discretized. An alternative approach consists in replacing the reinforced solid mass by a equivalent materiel made up by the superposition of several continuous media. Unlike utraditonal w theories of homogenisation, this method makes it possible to take into account different kinematics for the various continuous media, and thus to account for the relative slip between the ground and the inclusions. Moreover it is simpler and more rapid to be implemented.

The implementation of this model in the FEM software CESARLCPC have been undertaken. The current version of the code allows the elastoplastic analysis of two and three-dimensional problems, within the case where only traction and compression forces in inclusions are taken into account, and kinematic of each constitutive materials is supposed to be identical. The results obtained for the study of two reinforced works (analyzes three-dimensional compressing of a square foundation resting on piles, study of the convergence of a boited tunnel) are presented in this paper, showing the relevancy and the efficiency of the method. 


\section{1}

\section{Introduction}

L'utilisation d'inclusions possédant des propriétés mécaniques élevées pour renforcer des massifs de sol ou de roche est courante en géotechnique, par exemple pour assurer la stabilité du front de taille d'un tunnel ou celle d'un mur de soutènement.

Quel que soit l'ouvrage renforcé, ces inclusions ont généralement une géométrie linéaire (boulons ou clous métalliques, pieux en béton...) et sont souvent réparties de façon périodique avec des directions privilégiées. La dimension de leur section est petite devant celle caractérisant l'ouvrage étudié.

Pour le calcul de ces ouvrages, deux types de modélisation sont généralement mis en cuvre. Le premier consiste à tenir compte explicitement de la géométrie réelle de l'ouvrage et de ses inclusions. Le second vise à remplacer le matériau hétérogène constitué par le sol et les inclusions, par un matériau homogène équivalent. Cette approche présente l'avantage de simplifier la description géométrique de l'ouvrage, dans la mesure où il n'est plus nécessaire de mailler séparément les inclusions dans une discrétisation par éléments finis.

La méthode multiphasique (Sudret et de Buhan, 1999), que nous présentons dans la première partie de cet article, s'inscrit dans cette deuxième catégorie. Elle repose sur l'idée qu'en chaque point il y a coexistence d'un milieu représentant le massif avant renforcement et d'autant de milieux continus supplémentaires que de directions de renforcement. Dans ce cadre de modélisation, le principe des puissances virtuelles permet d'établir sans difficulté les équations d'équilibre. Les lois de comportement du matériau, dans le domaine élastoplastique, sont, quant à elles, décrites dans un cadre thermodynamique.

Dans la seconde partie, deux ouvrages typiques sont étudiés au moyen du progiciel CESAR-LCPC, dans lequel une version simplifiée du modèle théorique a été intégrée. Les résultats obtenus sont alors comparés à ceux obtenus par des méthodes classiques ou par voie expérimentale, mettant en évidence la pertinence de la méthode proposée.

\section{2}

\section{Modélisation multiphasique des milieux renforcés par inclusions}

Au cours des dernières années, le renforcement de sols ou de roches au moyen d'inclusions rigides s'est largement développé sous diverses formes (boulonnage, terre armée, etc.). La disposition spatiale des inclusions est en général périodique. Si le nombre de ces inclusions est suffisant, le recours à des méthodes d'homogénéisation parait naturel. Elles consistent à modéliser le milieu constitué de la matrice et des inclusions, comme un milieu homogène à l'échelle de l'ouvrage, le problème étant alors de relier ses propriétés à celles de la matrice et des inclusions. Ce type d'approche a déjà été mis en œuvre pour la construction de critères de résistance pour des matériaux à fibres (de Buhan et al., 1990, 1991), ainsi que pour la modélisation élastoplastique de tunnels boulonnés radialement (Greuell, 1993), et plus récemment pour le boulonnage du front de taille de tunnels (Jassionnesse, 1998).

L'approche proposée ici se caractérise par le fait que le milieu équivalent est un milieu homogène multi- phasique (Sudret 1999; de Buhan et Sudret, 1999; Sudret et de Buhan, 1999). Une version simplifiée du modèle a été introduite dans le progiciel CESAR-LCPC.

\section{1}

\section{Présentation du modèle}

On considère un matériau constitué d'un milieu continu dans lequel sont disposées des inclusions de renforcement linéaires orientées selon $\mathrm{N}$ directions différentes (Fig. 1).

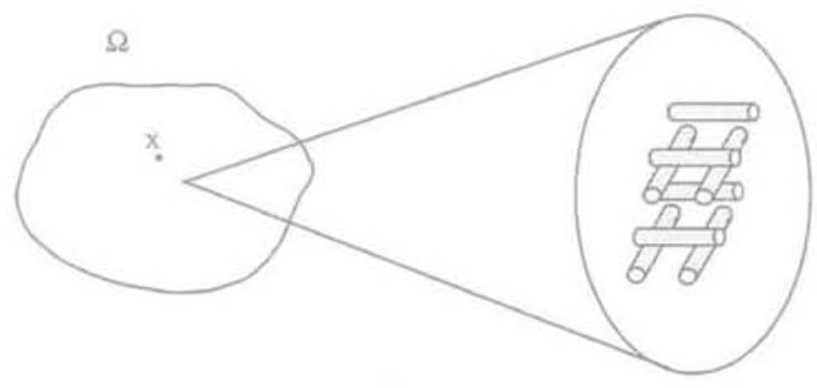

FiG. 1 Description géométrique du matériau renforcé (d'après Sudret, 1999).

Geometrical description of the reinforced material.

On fait l'hyptothèse qu'à l'échelle de l'ouvrage étudié (dite échelle macroscopique), le milieu peut être décrit comme un milieu continu multiphasique, c'està-dire comme la superposition de plusieurs milieux continus, associés à la matrice (sol ou roche) ou aux inclusions dans l'une des directions de renforcement. Cette hypothèse est à rapprocher de celle utilisée par Coussy (1991) pour décrire un milieu poreux. Dans la suite, les milieux continus constituant le milieu multiphasique seront appelés «phases».

Concrètement, la description du milieu propose qu'en un point de l'espace occupé par le milieu renforcé se trouvent plusieurs particules matérielles, animées chacune d'une cinématique propre. On notera $\underline{U}^{m}$ le champ de vitesse associé à la phase matrice, et $\underline{U} r$ ceux relatifs aux différentes phases de renforcement (Fig. 2). On note $\xi^{m}$ et $\xi^{r}$ les champs de déplacement associés aux phasés matrice et renforcement.
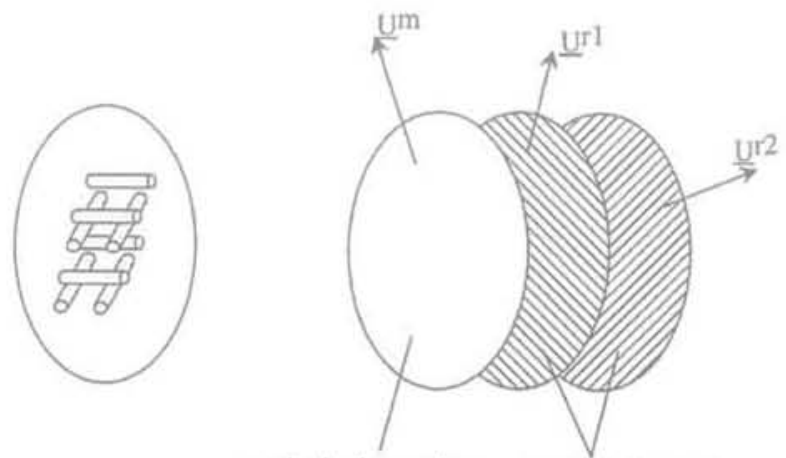

particule de matrice

particule de renforcement

milieu renforcé réel

modèle multiphasique

FIG. 2 Description cinématique du milieu multiphasique; cas de deux directions de renforcement.

Kinematical description of the multiphase model; cas of two reinforced directions. 


\section{Efforts intérieurs. Équations d'équilibre}

La construction des efforts par le principe des puissances virtuelles conduit à définir des efforts intérieurs dans chacune des phases du milieu multiphasique. Ils ne sont pas de la même forme selon la phase considérée. Dans la phase associée à la matrice, ils sont représentés par un tenseur symétrique du second ordre noté $\underline{\sigma}^{m}$, que l'on appellera tenseur des contraintes partielles dans la matrice. Le tenseur des efforts associés à chaque phase de renforcement est, quant à lui, uniaxial, compte tenu du caractère curviligne des inclusions. Il sera donc de la forme $\sigma^{r}=\sigma^{r} e \otimes e$, où $e$ est le vecteur unitaire dans la direction des inclusions de la phase considérée et $\sigma^{r}$ est un scalaire représentant la contrainte partielle dans ces inclusions, dont on précisera le sens physique ultérieurement.

Le principe des puissances virtuelles conduit à l'expression suivante des équations d'équilibre de chacune des phases:

$$
\operatorname{div} \underline{\underline{\sigma}}^{m}+\rho^{m}\left(\underline{F}^{m}-\underline{\gamma}^{m}\right)+\sum_{r=1}^{N} \underline{I}^{r}=0 \text { pour la phase matrice }
$$

$\operatorname{div} \underline{\underline{\sigma}}^{r}+\rho^{r}\left(\underline{F}^{r}-\underline{\gamma}^{r}\right)-\underline{I}^{r}=0(r=1, \ldots, N)$ pour la phase renforcement

où $\rho^{m}, E^{m}$ et $\gamma^{m}$ (resp., $\rho^{r}, E^{r}$ et $\gamma^{r}$ ) désignent la masse volumique, la densité volumique d'efforts extérieurs et l'accélération de la phase matrice (resp. de la phase de renforcement $r$ ) et $I$ la densité volumique de force d'interaction exercée par la phase de renforcement $r$ sur la phase matrice. On notera que cette modélisation ne peut prendre en compte d'éventuelles interactions entre deux phases de renforcement. Cette restriction est en fait sans conséquence, étant donnée l'absence de contact entre les inclusions dans deux directions différentes.

Précisons maintenant le sens physique de la contrainte partielle $\sigma$, relative à la phase renforcement. Pour cela, définissons le système matériel $\Omega$ constitué du milieu renforcé, et $\Omega^{\prime}$ un sous-sytème quelconque de $\Omega$; en tout point $\chi$ de la frontière $\partial \Omega^{\prime}$ et de $\Omega^{\prime}$, les densités surfaciques d'efforts $\underline{T}^{n}$ et $T$ exercées par l'extérieur de $\Omega^{\prime}$ à travers $\partial \Omega^{\prime}$ sur la phase matrice et les différentes phases de renforcement sont données par:

$\underline{T}^{m}(\underline{x})=\underline{\sigma}^{m}(\underline{x}) \cdot \underline{n}(\underline{x})$ pour la phase matrice

$\underline{T}^{r}(\underline{x})=\sigma^{r}(\underline{x})\left(\underline{n}(\underline{x}) \cdot \underline{e}_{r}\right) \cdot \underline{e}_{r}(r=1, \ldots, N)$ pour les phases renforcement (4)

où $\underline{n}(\mathrm{x})$ est la normale extérieure à $\Omega^{\prime}$ au point $\underline{x}$ et $T^{m}$.

On peut dès lors proposer une interprétation simple de la contrainte partielle $\sigma^{r}$ dans la phase de renforcement $r$. En effet, la résultante des efforts extérieurs s'exerçant sur la phase $r$ à travers un élément de surface $\underline{d S}$ de normale $\underline{\underline{n}}$ et d'arc $d S$ s'écrit d'après (4):

$$
\underline{F}^{r}=\underline{T}^{r} \cdot d S=\sigma^{r}\left(\underline{\underline{n}} \cdot \underline{e}_{r}\right) d S \underline{e}_{r}
$$

On remarque que $\left(\underline{n}\right.$. $\left.\underline{e}_{\mathbf{T}}\right) d S$ est l'aire de la projection de l'élément de surface $\underline{d S}$ sur un plan perpendiculaire à la direction de renforcement. D'autre part, la force élémentaire $F^{\prime} s^{\prime}$ identifie naturellement au produit de la contrainte moyenne $\sigma_{\text {inc }}$ dans les inclusions avec la fraction $s_{\text {in }}$ de surface effectivement coupée par les inclusions à l'échelle microscopique dans un plan perpendiculaire à la direction des inclusion (Fig. 3). En constatant que $\sigma_{\text {inc }}$ est égale à la fraction volumique $\eta^{r}$ des inclusions dans le milieu renforcé, on aboutit à la relation entre la contrainte moyenne dans les inclusions $\sigma_{i n c}$ et la contrainte partielle $\sigma^{\prime}$ dans la phase $r$ du milieu multiphasique:

$$
\sigma_{i n c}=\sigma^{r} / \eta^{r}
$$
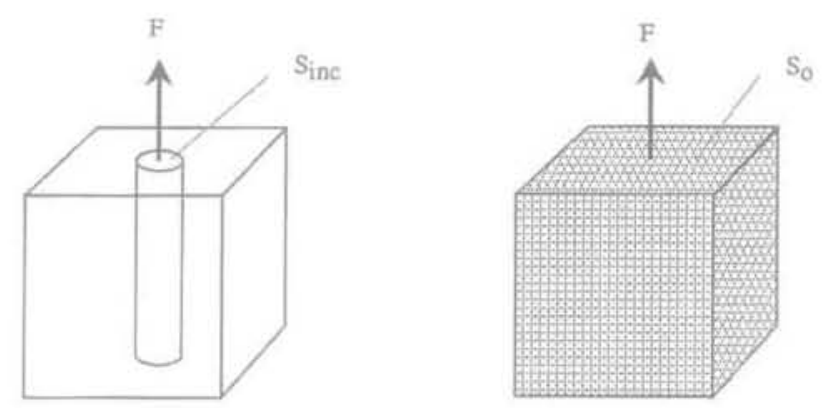

a- effort sur une inclusion dans un volume elementaire du milieu renforce $\mathrm{F}=\sigma_{\text {inc }} s_{\text {inc }}$

$$
\begin{aligned}
& \text { b- effort sur une phase } \\
& \text { renforcement du } \\
& \text { milieu multiphasique } \\
& F=\sigma^{r} s_{0}
\end{aligned}
$$

FG.3 Interprétation physique de la contrainte partielle $\sigma$.

Physical interpretation of the partiai stress $\sigma$.

\section{2,3}

\section{Formulation du comportement élastique}

Nous nous proposons maintenant de formuler les relations de comportement du milieu multiphasique. Les deux premiers principes de la thermodynamique, généralisant l'inégalité de Clausius-Duhem aux milieux multiphasiques, conduisent à l'inégalité suivante:

$\left.\left(\sigma^{m}-\frac{\partial \psi^{m}}{\partial \underline{\varepsilon}^{m}}\right) \cdot \xi^{m}+\sum_{r=1}^{N}\left(\sigma^{r}-\frac{\partial \psi^{r}}{\partial e^{r}}\right) \cdot \xi^{r}+\sum_{r=1}^{N}\left(1^{r}-\frac{\partial \psi^{L r}}{\partial\left(\underline{\xi}^{r}-\underline{\xi}^{m}\right.}\right)\right):\left(\xi^{r}-\underline{\xi}^{m}\right) \geq 0(7)$ où $\varepsilon^{m}=\frac{1}{2}\left(\underline{\underline{\operatorname{grad}} \xi^{m}}+\underline{\left.\underline{\operatorname{grad}} \xi^{m}\right)}\right.$ désigne le tenseur linéarisê de la phase matrice, $\varepsilon^{r}=\left(\underline{\underline{\operatorname{grad}} \underline{\xi}^{r}} \cdot \underline{e}_{r}\right) \cdot \underline{e}_{r}$ la déformation de la phase $r$ dans la direction des inclusions et $\left(\xi^{r}-\xi^{m}\right)$, le déplacment relatif de la phase $r$ par rapport à la phase matrice, L'inégalité (7) est une condition nécessaire que doivent vérifier les lois de comportement du milieu multiphasique. Pour le cas particulier de l'élasticité, l'inégalité se transforme en égalité, correspondant à un milieu réversible du milieu multiphasique. On fait maintenant I'hypothèse que l'énergie libre $\psi$ du mulieu multiphasique se décompose de la manière suivante:

$$
\psi\left(\underline{\varepsilon}^{m}, \varepsilon^{r}, \underline{\xi}^{r}-\underline{\xi}^{m}\right)=\psi^{m}\left(\underline{\underline{\varepsilon}} \underline{\underline{\varepsilon}}^{m}\right)+\sum_{r=1}^{N} \psi^{r}\left(\varepsilon^{r}\right)+\sum_{r=1}^{N} \psi^{L r}\left(\underline{\xi^{r}}-\underline{\xi}^{m}\right)
$$

Si l'on admet de plus l'indépendance des variables $\varepsilon^{m}, \varepsilon^{r}$ et $\left(\xi^{r}-\xi^{m}\right)$, le comportement est cararctérisé par les relations suivantes:

$$
\begin{gathered}
\underline{\sigma}^{m}=\frac{\partial \psi^{m}}{\partial \underline{\varepsilon}^{m}} \\
\sigma^{r}=\frac{\partial \psi^{r}}{\partial \varepsilon^{r}} \quad r=1, \ldots, N \\
\underline{I}^{r}=\frac{\partial \psi^{l, r}}{\partial\left(\underline{\underline{\xi}}^{r}-\underline{\xi}^{m}\right)} \quad r=1, \ldots, N
\end{gathered}
$$




\section{Comportement élastoplastique adhérent}

Pour les problèmes de géotechnique il est évidemment nécessaire de définir une loi de comportement élastoplastique du matériau multiphasique. Une première formulation peut être obtenue en considérant une description plus simple de la cinématique du milieu multiphasique, conduisant à faire l'hypothèse d'adhérence parfaite entre les différentes phases (de Buhan et Sudret, 1999).

\subsection{1.}

\section{Hypothèse d'adhérence parfaite}

Cette hypothèse consiste à considérer des champs de déplacements identiques dans toutes les phases. On note $\xi(x)$ le champ commun à toutes les phases. Il est important à ce stade d'attirer l'attention du lecteur sur la portée d'une telle hypothèse. Celle-ci se rapporte directement sur la modélisation multiphasique. Elle n'implique donc nullement une condition de non-glissement à l'interface entre les inclusions et la matrice à l'échelle réelle. Cette hypothèse ne nous intercit donc pas de traiter en première approximation le cas d'un matériau dans lequel peuvent se produire des décohésions locales d'interface.

Écrivons maintenant ce que devienne les équations d'équilibre régissant le milieu multiphasique dans le cas de l'adhérence parfaite. Notons $\underline{\underline{\varepsilon}}=\frac{1}{2}(\underline{\underline{\operatorname{grad} \xi}}+\underline{\underline{\operatorname{grad} \xi}})$ le tenseur des déformations associê au champ $\xi$, il vient:

$$
\underline{\underline{\varepsilon}}^{m}=\underline{\underline{\varepsilon}} ; \varepsilon^{r}=\underline{\underline{\varepsilon}}:\left(\underline{e}_{r} \otimes \underline{e}_{r}\right)
$$

Dans la mesure où le déplacement relatif $\xi^{m}-\xi^{r}$ est nul, il n'est plus nécessaire de modéliser lés forces d'interaction Ir entre la phase matrice et les phases renforcement. On élimine ces efforts en combinant les équations d'équilibre (1) et (2), on obtient alors l'équation d'équilibre globale suivante:

$$
\operatorname{div} \underline{\underline{\Sigma}}+p^{m}\left(\underline{F}^{m}-\underline{\gamma}^{m}\right)+\sum_{r=1}^{N} p^{r}\left(\underline{F}^{r}-\underline{\gamma}^{r}\right)=0
$$

où $\Sigma$ désigne le tenseur des contraintes totales, ayant poữ expression:

$$
\underline{\underline{\Sigma}}=\underline{\underline{\sigma}}^{m}+\sum_{r=1}^{N} \sigma^{r} \underline{e}_{r} \otimes \underline{e}_{r}
$$

Une autre conséquence de l'hypothèse d'adhérence parfaite concerne la forme des conditions aux limites: les cinématiques des phases étant identiques, il n'est plus possible d'appliquer indépendamment des efforts sur chacune des phases à travers une surface géométrique quelconque. Les conditions limites en effort définies en un point $\underline{x}$ de la frontière du domaine $\Omega$ s'écrivent maintenant:

$$
\underline{T}(\underline{x})=\underline{\underline{\Sigma}}(\underline{x}) \cdot \underline{n}(\underline{x})
$$

\section{492}

\section{Formulation du comportement élastoplastique}

La formulation élastoplastique proposée par Sudret (1999) pour le milieu adhérent consiste à adopter pour chaque phase un comportement élastique linéaire parfaitement plastique avec une règle d'écoulement asso- ciée. La déformation de la phase matrice et des phases renforcement est décomposée en deux contributions élastique et plastique :

$$
\begin{gathered}
\underline{\varepsilon}^{m}=\underline{\underline{\varepsilon}}_{\underline{p}}^{m}+{\underline{\underline{\varepsilon_{p}}}}_{p}^{m} \\
\varepsilon^{r}=\varepsilon_{e}^{r}+\varepsilon_{p}^{r} \quad r=1, \ldots, \mathrm{N}
\end{gathered}
$$

où les indices e et $p$ désignent respectivement les parties élastique et plastique des déformations. Le comportement s'écrit alors:

$$
\begin{gathered}
\stackrel{\underline{\sigma}^{m}}{=} \underline{\underline{\underline{a}}}^{m}:\left(\underline{\underline{\varepsilon^{m}}}-\underline{\underline{\varepsilon^{m}}}\right) \\
\sigma^{r}=a^{r}\left(\varepsilon^{r}-\varepsilon_{p}^{r}\right) \quad r=1, \ldots, \mathrm{N} \\
\dot{\mathrm{E}}_{=p}^{m}=\lambda^{m} \frac{\partial f^{m}}{\partial \underline{\underline{\sigma}}^{m}} \operatorname{avec} \lambda^{m} \geq 0 \\
\dot{\varepsilon}_{p}^{r}=\lambda^{r} \frac{\partial f^{r}}{\partial \underline{\sigma}^{r}} \operatorname{avec} \lambda^{r} \geq 0 \quad r=1, \ldots, N
\end{gathered}
$$

où $\mathrm{a}^{\mathrm{tm}}$ est le tenseur des modules élastiques de la phase matrice, a la raideur de la phase renforcement $r, f^{\prime \prime \prime}\left(\sigma^{m}\right)$ le critère de plasticité de la matrice, et $f^{t}\left(\sigma^{r}\right)$ une foñction critère qui définit le domaine des valeurs de $\sigma^{r}$ pour lesquelles la déformation plastique dans la phase $r$ n'évolue pas. Si la phase concernée reste élastique tant que $\sigma^{r}$ est dans l'intervalle $\left[-\sigma^{\prime}, \sigma^{\prime}\right]$ la fonction $f^{\prime}\left(\sigma^{\prime}\right)$ s'écrit:

$$
f^{r}\left(\sigma^{r}\right)=\max \left(-\sigma^{r}-\sigma_{-}^{r}, \sigma^{r}-\sigma_{+}^{r}\right)
$$

On considérera, en général, que la résistance d’une phase de renforcement est plus faible en compression qu'en traction, en raison de la possibilité de flambement des inclusions.

La formulation ci-dessus appelle trois remarques:

- Rapprochant (14) de (18) et (19), il apparait qu'en l'absence de déformations plastiques dans la matrice et les phases de renforcement la contrainte totale dans le milieu renforcé est reliée à la déformation $\underline{\underline{\varepsilon}}$ par:

$$
\underline{\underline{\underline{\Sigma}}}=\underline{\underline{\underline{\underline{\underline{A}}}}}: \underline{\underline{\varepsilon}}
$$

où le tenseur $\underline{\underline{\underline{\underline{A}}}}$ est défini par:

$$
\underline{\underline{\underline{\underline{A}}}}=\underline{\underline{a}}_{\underline{\underline{E}}}^{m}+\sum_{r=1}^{N} a^{r} \underline{e}_{r} \otimes \underline{e}_{r} \otimes \underline{e}_{r} \otimes \underline{e}_{r}
$$

Autrement dit, la présence de renforcement se traduit par une modification des modules d'élasticité reliant la déformation du milieu multiphasique aux contraintes totales. Dans le cas où l'élasticité de la matrice est isotrope, la présence d'inclusions se traduit par une anisotropie du tenseur d'elasticité global $\underline{\underline{\underline{\underline{A}}}}$.

On notera que la loi élastique définie par (23) et (24) est conforme au résultat d'une démarche d'homogénéisation élastique classique (Greuell, 1993).

- Pour certains types de matériaux, il peut être préférable d'adopter pour la matrice une loi d'écoulement non associée. La relation (20) est alors remplacée par:

$$
\underline{\dot{\varepsilon}}_{=p}^{m}=\lambda^{m} \frac{\partial g^{m}}{\partial \underline{\underline{\sigma}}^{m}} \text { avec } \lambda^{m} \geq 0
$$

où $g^{m}\left(\underline{\underline{\sigma}}^{m}\right)$ est le potentiel plastique.

- Enfin, il est intéressant de noter que bien que chacune des phases ait un comportement élastique parfai- 
tement plastique, le comportement du milieu multiphasique est écrouissable. Cette remarque constitue l'un des rapports importants de la méthode multiphasique. Les méthodes d'homogénéisation classique ne possèdent pas une telle propriété.

\section{3}

\section{Liens avec les propriétés des constituants}

Il reste à identifier les paramètres qui décrivent le comportement des différentes phases (modules élastiques $\underline{a}^{m}, a^{r}$ et critères de plasticité $\left.f^{m}, f^{\prime}\right)$. Cette identification est ici abordée de manière phénoménologique: la démarche n'est pas orientée vers une identification à partir des propriétés des constituants par une méthode de changement d'échelle ou d'homogênéisation.

En pratique, il est naturel de modéliser le comportement de la phase matrice de la même manière que celui cuu milieu continu dans lequel sont placées les inclusions. Pour ce qui concerne les phases de renforcement, un raisonnement simple (analogue à celui mené en 2.2) conduit à identifier la raideur d'une phase de renforcement au produit du module de Young des inclusions correspondantes par leur fraction volumique:

$$
a^{r}=\eta^{r} E_{i n c}^{r}
$$

\section{n94.4.}

\section{Mise en œuvre numérique}

La méthode multiphasique, décrite ci-dessus et mise en ceuvre par Sudret et al. (2000) dans le cadre d'un code de calcul prototype, a été introduite dans le progiciel CESAR-LCPC. Pour le moment, la version intégrée repose sur l'hypothèse d'adhérence parfaite entre les phases, c'est-à-dire qu'elle prend en compte un champ de déplacement identique pour toutes les phases matrice et renforcement. On a pu ainsi réaliser des études sur deux types d'ouvrages renforcés :

- la première concerne le comportement de radiers de pieux. Les résultats obtenus par notre approche sont confrontés à ceux obtenus par une méthode classique de dimensionnement de ce type d'ouvrages;

- la seconde étude est relative à la convergence d'une galerie souterraine boulonnée radialement.

\section{3}

\section{Mise en œuvre de la méthode sur des ouvrages de géotechnique}

\section{1}

\section{Calcul de tassement de radiers de pieux}

\subsection{4}

\section{Description du problème}

On s'intéresse à un radier carré de côté $\mathrm{B}=36 \mathrm{~m}$ et d'épaisseur $\mathrm{e}=5 \mathrm{~m}$, reposant sur un massif de sol renforcé par un réseau de $9 \times 9$ pieux de diamètre $\mathrm{d}=0,8 \mathrm{~m}$, de longueur $\mathrm{L}=20 \mathrm{~m}$, espacés d'une distance $\mathrm{s}=4 \mathrm{~m}$ de centre à centre (Fig. 4).
B

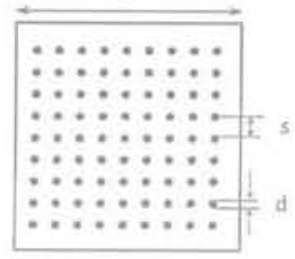

FIG.4 Description géométrique du radier de pieux.

Geometrical description of the piled raft foundation.

Le massif est constitué d'un sol homogène élastique de module de Young $\mathrm{E}$, et de coefficient de Poisson v. Le radier et les pieux sont construits en béton de modules de Young respectifs $\mathrm{E}_{r}$ et $\mathrm{E}_{n}$ égaux à $35 \mathrm{GPa}$. Le tableau I récapitule les données géométriques et mécaniques du problème.

TABLEAU1 Caractéristiques du problème. Parameters of the problem.

\begin{tabular}{l|l|l}
\multicolumn{1}{c|}{ Massif de sol } & \multicolumn{1}{|c|}{ Radier } & \multicolumn{1}{c}{ Pieux } \\
\hline$E_{s}=280 \mathrm{MPa}$ & $\mathrm{E}_{r}=35 \mathrm{GPa}$ & $\mathrm{E}_{\mathrm{p}}=35 \mathrm{GPa}$ \\
\hline $\mathrm{v}_{\mathrm{y}}=0,4$ & $\mathrm{v}_{r}=0,3$ & $\mathrm{~L}=20 \mathrm{~m}$ \\
\hline & $\mathrm{e}=5 \mathrm{~m}$ & $\mathrm{~d}=0,8 \mathrm{~m}$ \\
\hline & $\mathrm{B}=36 \mathrm{~m}$ & $\mathrm{~s}=4 \mathrm{~m}$ \\
\hline
\end{tabular}

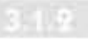

\section{Modélisation numérique}

Cette simulation est réalisée dans le cadre d'une modélisation tridimensionnelle. Compte tenu des symétries du problème, nous n'étudions qu'un huitième de la géométrie de l'ouvrage (cf. maillage Figs. 5 et 6). Étant donné la valeur élevée du coefficient de Poisson du sol, nous avons été amenés à mailler une zone relativement grande vis-à-vis de la dimension du radier $(30 \mathrm{~B} \times 30 \mathrm{~B} \times$ 30B), afin d'éviter les effets de bord.

Le maillage est constitué de 4200 pentaèdres à 15 nœuds, et compte 12500 nceuds. Il est séparé en trois groupes: un groupe relatif au radier, un autre représentant la zone renforcée et le troisième discrétisant le reste du massif (Fig. 7).

En ce qui concerne les conditions limites en déplacement, elles portent sur les quatre surfaces suivantes: - surface inférieure $\mathrm{z}=-15 \mathrm{~B}$ : déplacements verticaux bloqués

- surfaces verticales $x=15 B, y=15 B, x+y=15 B$ : déplacements bloqués perpendiculairement aux surfaces.

\section{6}

\section{Modèle hybride (Griffiths et al., 1991)}

Le problème du comportement de radier de pieux a été étudié par Clancy et al. (1993), qui ont mis au point une technique de calculs simplifiée basée sur la méthode hybride proposée par Chow (1986). Elle repose sur les principales hypothèses suivantes: 


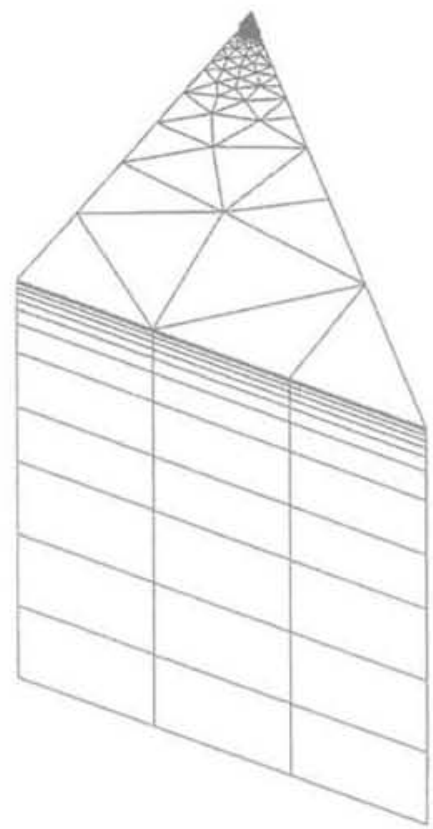

Maillage utilisé pour l'étude. Mesh used for the calculations.

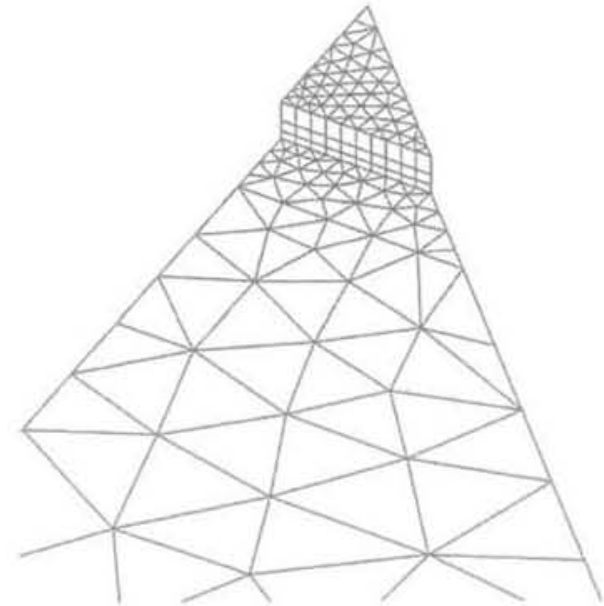

คG. 6 Vue agrandie du maillage. Magnified mesh view.

- les pieux sont discrétisés en éléments de barre ne travaillant qu'en traction compression. Le radier est modélisé par des éléments finis de plaque en flexion; - en réponse à un chargement vertical, seuls les déplacements verticaux sont pris en compte;

- l'interaction entre les pieux et le sol est modélisée par des ressorts relatifs aux efforts de cisaillement induits dans le sol (Randolph et Wroth, 1978; Kraft et al., 1981). La réaction du sol sous le radier est modélisée par des ressorts dont la raideur est obtenue par la solution de Giroud (1968). L'interaction entre le radier et les pieux est prise en compte en utilisant la solution de Mindlin.

Ces hypothèses étant précisées, on définit la rigidité $k_{p r}$ d'un radier de pieux construit dans un massif de sol élastique, comme le rapport de la résultante $P$ des efforts appliqués au tassement moyen:

$$
k_{p r}=\frac{P}{W_{\text {moyen }}}=\frac{P_{p}+P_{r}}{W_{\text {moyen }}}
$$

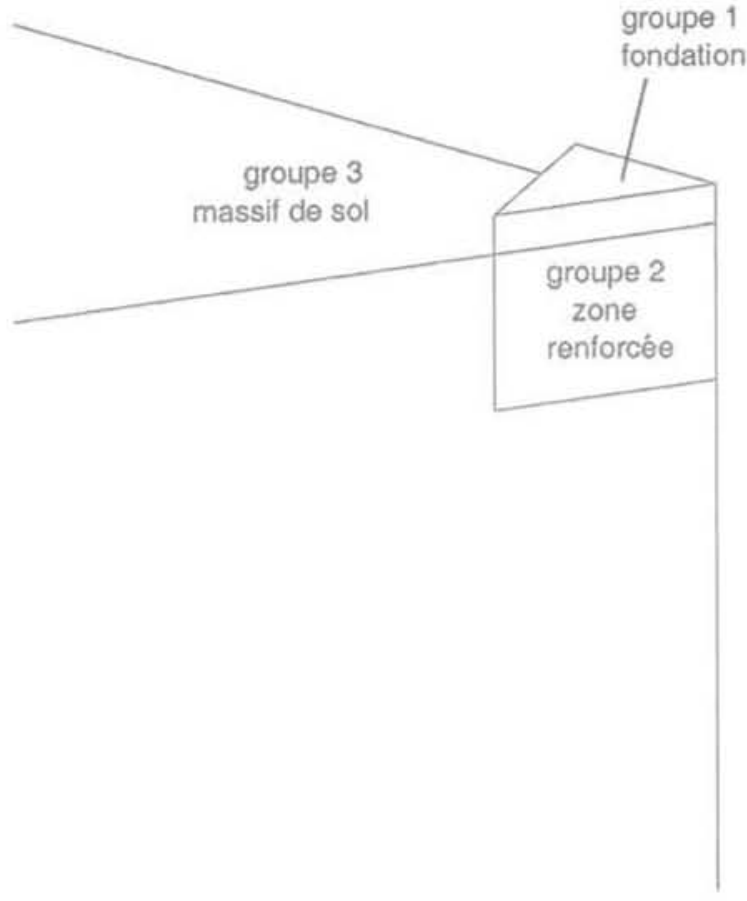

FG. 7 Groupes d'éléments correspondant aux zones de mêmes caractéristiques mécaniques.

Elements groups related to areas of identical mechanical properties.

où $P_{r}$ et $P_{r}$ sont les proportions respectives de la résultante $\mathrm{P}$ appliquée sur les pieux et sur le radier.

\subsection{4}

\section{Comparaison des deux approches}

Les valeurs numériques, calculées par les deux approches, des différentes quantités caractérisant le radier de pieux introduites précédemment sont reportées dans le tableau II.

On constate une bonne concordance entre les deux approches, les écarts étant inférieurs à $10 \%$. Le modèle multiphasique semble conduire à un comportement plus raide de la structure. Ceci peut notamment être expliqué par le fait que le modèle aux éléments finis est borné par des frontières rigides à une distance finie $(30 \times B)$, alors que le modèle hybride fait intervenir le caractère semi-infini du massif. De plus, nous rappelons que le calcul a été effectué avec l'hypothèse d'adhérence parfaite entre les phases, ce qui est évidernment de nature à "renforcer » le matériau équivalent. Un calcul avec prise en compte de cinématiques différentes pour chaque phase conduirait naturelle-

TABLEAUII Comparaison des résultats numériques. Comparison of numerical results.

\begin{tabular}{l|l|l} 
& \multicolumn{1}{|c|}{ Clancy } & $\begin{array}{c}\text { Méthode } \\
\text { multiphasique }\end{array}$ \\
\hline$w$ (tassement max) & $84 \mathrm{~mm}$ & $76 \mathrm{~mm}$ \\
\hline$P_{p}$ & $868 \mathrm{MN}$ & $810 \mathrm{MN}$ \\
\hline$P_{r}$ & $428 \mathrm{MN}$ & $389 \mathrm{MN}$ \\
\hline$k_{p r}$ & $15,42 \mathrm{MN} \cdot \mathrm{m}^{-1}$ & $15,78 \mathrm{~m}^{-1}$ \\
\hline
\end{tabular}


ment à un radoucissement du comportement d'ensemble.

La figure 9 trace l'évolution du tassement normalisé sous la fondation défini par Clancy de la manière suivante:

$$
\bar{W}=\frac{W-W_{\text {mayen }}}{W_{\max }-W_{\text {mayen }}}
$$

en fonction de la coordonnée normalisée $\lambda$, valant 0 au coin du radier, croissant linéairement jusqu'à 0,5 en suivant le côté, puis jusqu'à 1 en direction du centre (voir Fig. 8).

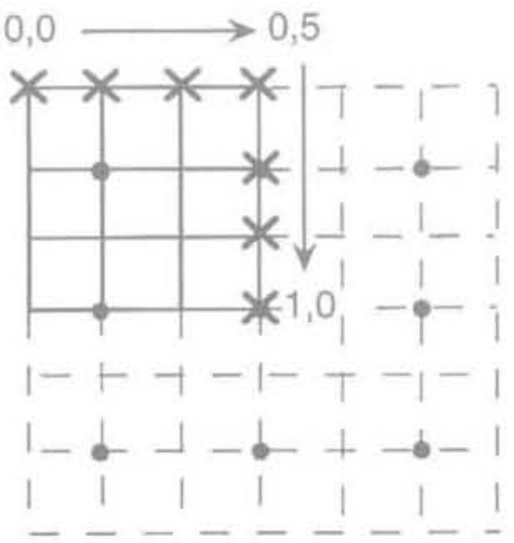

FIG. Coordonnée normalisée (d'après Clancy et Randolph).

Normalized coordinate (from Clancy and Randolph).

On constate ici encore une très bonne concordance des résultats des deux approches. II faut noter, en outre, que les temps de calculs d'un tel ouvrage prennent une trentaine de minutes, contre plus d'une dizaine d'heures avec la méthode hybride.

\section{2}

\section{Calcul de la convergence d'une galerie}

Après avoir étudié une structure tridimensionnelle, on s'intéresse maintenant à la convergence des parois d'un tunnel, que l'on aborde dans une approche bidimensionnelle en déformation plane, dans un plan perpendiculaire à l'axe de la galerie. Le problème consiste à évaluer la réduction des déplacements de la paroi que l'on peut obtenir en boulonnant le terrain perpendiculairement à la paroi de la galerie.

\section{9 .1}

\section{Description du problème}

On considère donc une galerie de section non circulaire dont la voûte et les piédroits sont renforcés par un boulonnage radial. Étant donné la symétrie du problème par rapport au plan vertical contenant l'axe du tunnel, seule une moitié de l'ouvrage sera étudiée (Fig. 10).

La voûte, le piédroit et le radier du tunnel sont circulaires : la première est constituée d'un quart de cercle de centre $(0,0)$ et de rayon $5 \mathrm{~m}$, les deux derniers d'arcs de cercle de centres respectifs $(-5,0),(0,5)$ et de rayon $10 \mathrm{~m}$.

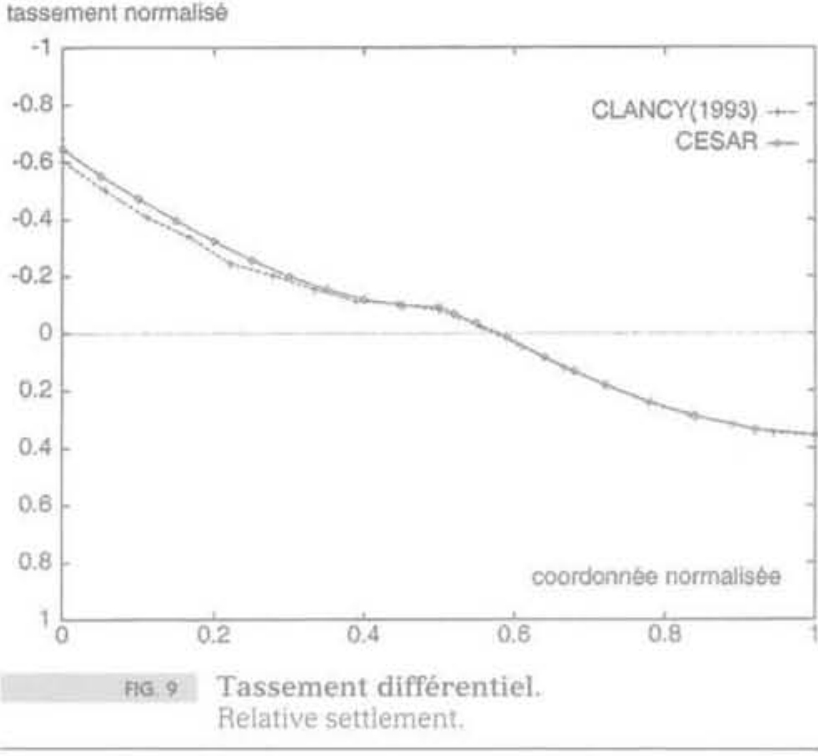

Le sol est constitué d'un matériau frottant de module de Young $50 \mathrm{MPa}$, de coefficient de Poisson 0,4, de cohésion $150 \mathrm{kPa}$ et d'angle de frottement et de dilatance égaux à $25^{\circ}$; il est supposé obéir à un critère de Drucker Prager. Les boulons, constitués d'acier $(E=200000 \mathrm{MPa})$, ont une longueur de $3,75 \mathrm{~m}$ et une section circulaire de diamètre $25 \mathrm{~mm}$. Leur densité en paroi est de 1 boulon $/ 2,5 \mathrm{~m}^{2}$.

Les éléments du maillage sont rassemblés en trois groupes (Fig. 10) : le groupe 1 correspond à la zone renforcée entourant la voûte, le deuxième à celle entourant le piédroit, le dernier groupe décrivant le reste du massif non renforcé.

La zone maillée est un cadre rectangulaire ayant pour largeur $50 \mathrm{~m}$ et pour hauteur $100 \mathrm{~m}$. Le maillage est constitué de 454 éléments (65 quadrilatères à 8 nouds et 389 triangles à 6 nouds) contenant 1029 nœuds (Fig. 11).

Le chargement est défini par une densité de forces d'excavation $p_{\text {c }}$ calculées à partir d'un champ de contraintes initial géostatique. On applique le déconfinement en attribuant un facteur multiplicatif $1-\lambda$ à $p$, $\lambda$ allant de 0 (déconfinement total), à 1 (pas de déconfinement).

\section{8}

\section{Résultats}

Nous présentons dans un premier temps le champ de contraintes (totales) (Fig. 12). Ce champ est conforme à notre attente, et l'on note au passage que l'on respecte bien la condition de contrainte libre sur les parois du tunnel.

Les figures 13 et 14 décrivent respectivement la forme et les isovaleurs du champ de contraintes partielles dans la phase de renforcement. Ces contraintes sont unidirectionnelles et suivent les directions de boulons. Il est à noter en outre, que la contrainte dans la phase de renforcement diminue lorsque l'on s'éloigne de la paroi et devient très faible en limite extérieure de zone renforcée. Ceci permet ainsi une discussion sur la longueur efficace des boulons en fonction des différents paramètres. En revanche, on observe qu'elles ne s'annulent pas 


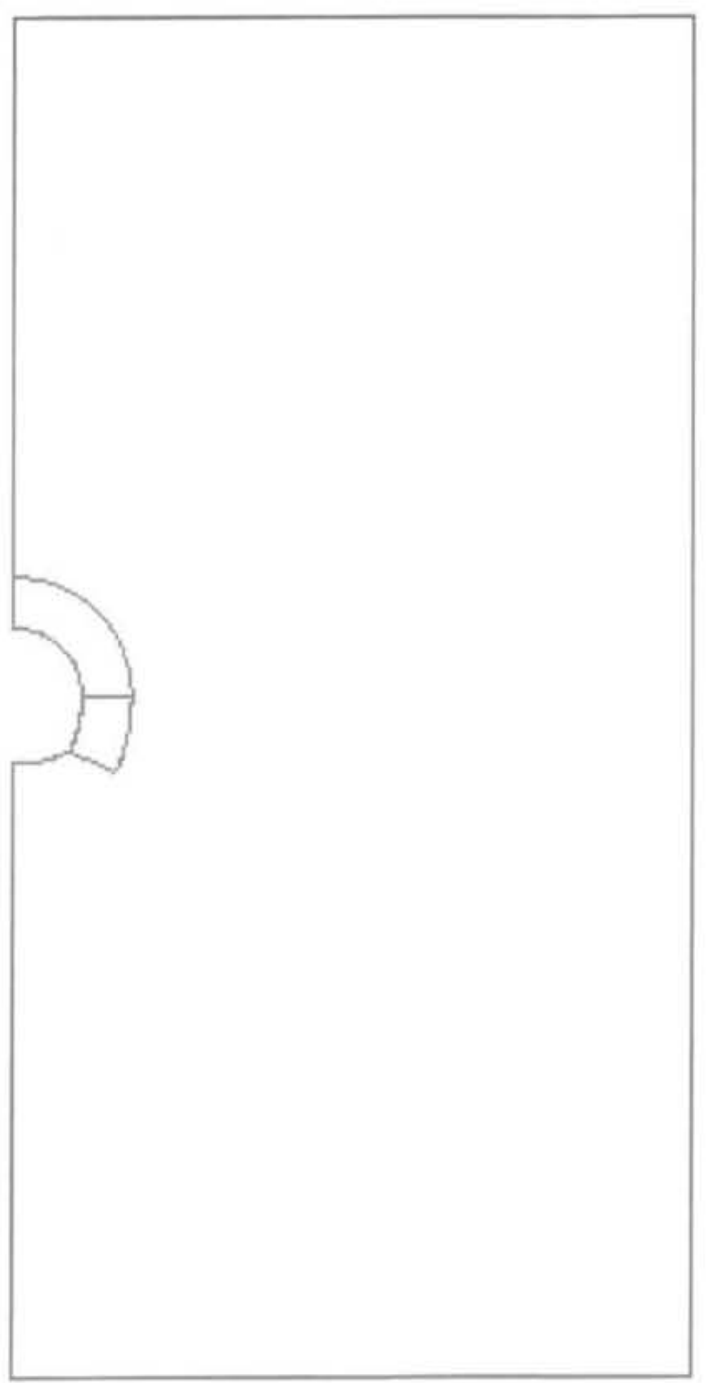

FG. 10 Tunnel renforcé par boulonnage radial de la voûte et du piédroit.

Tunnel reinforced by perpendicular bolts.

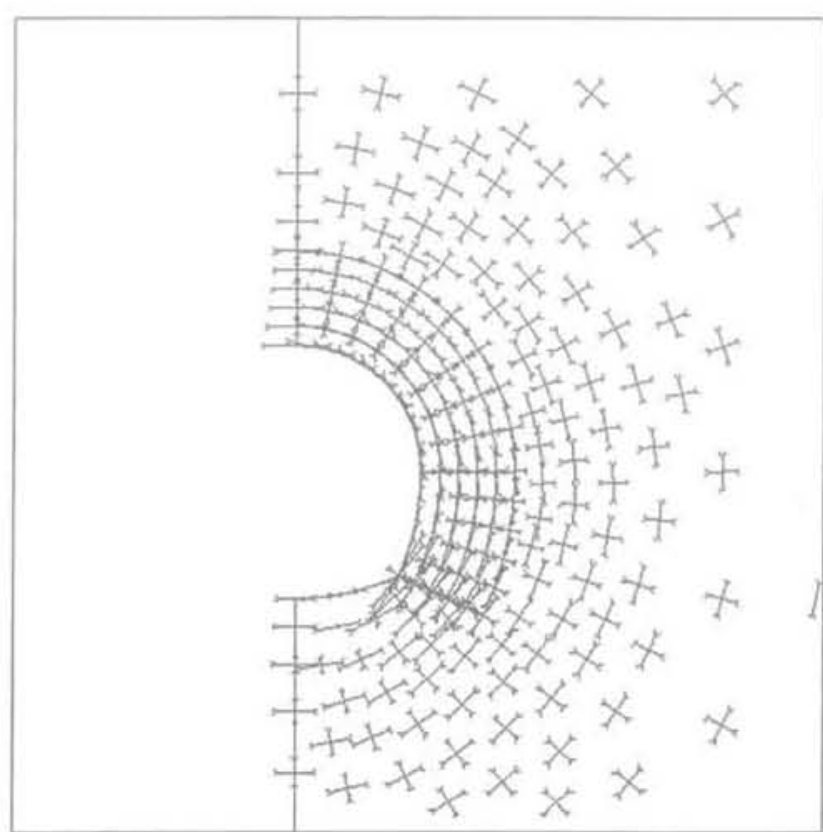

FG. 12 Champ de contraintes totales principales. Principal total stress field.

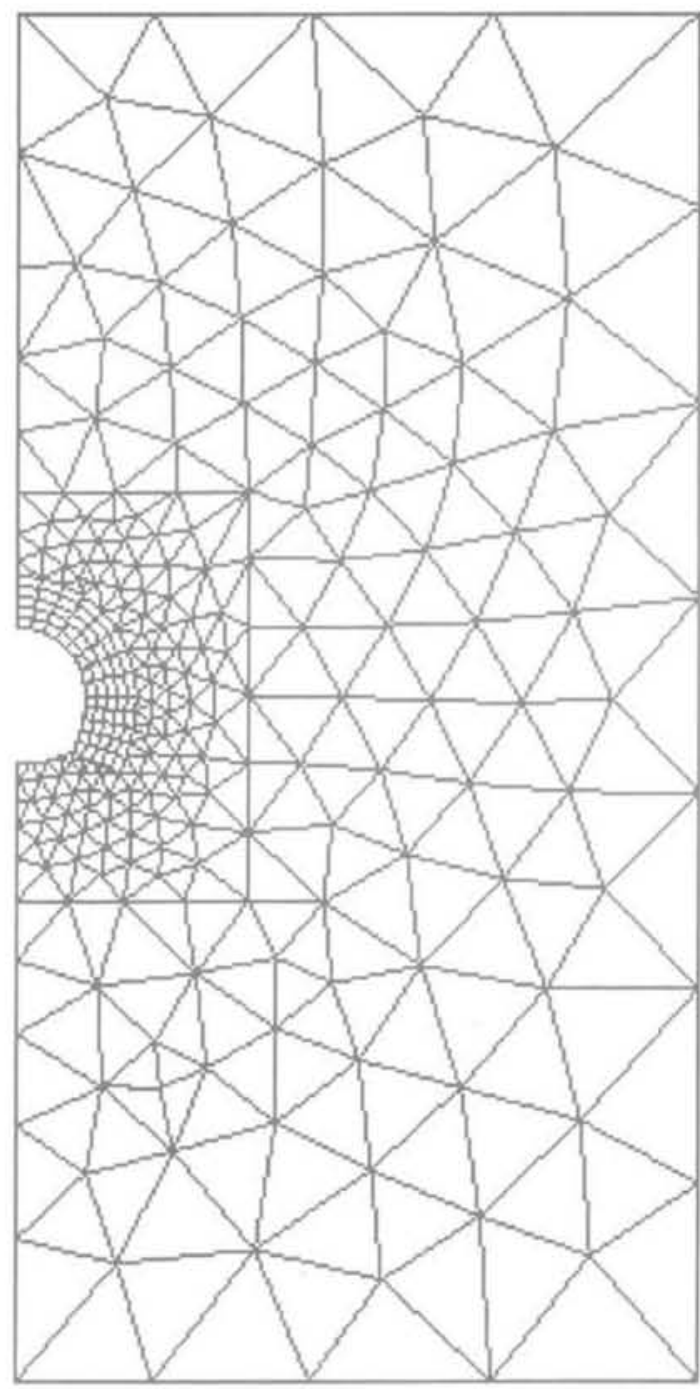

FIG 11 Discrétisation de l'ouvrage. Meshing of the ground around the tunnel.

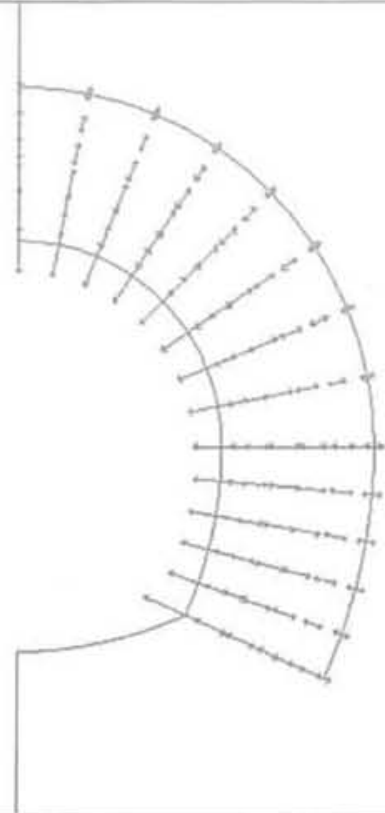

FIG. 13 Champ de contraintes $\sigma^{r}=\sigma^{r} e_{r} \otimes \underline{e}_{r}$ dans la phase de renforcement. Stress field in the reinforced phase. 


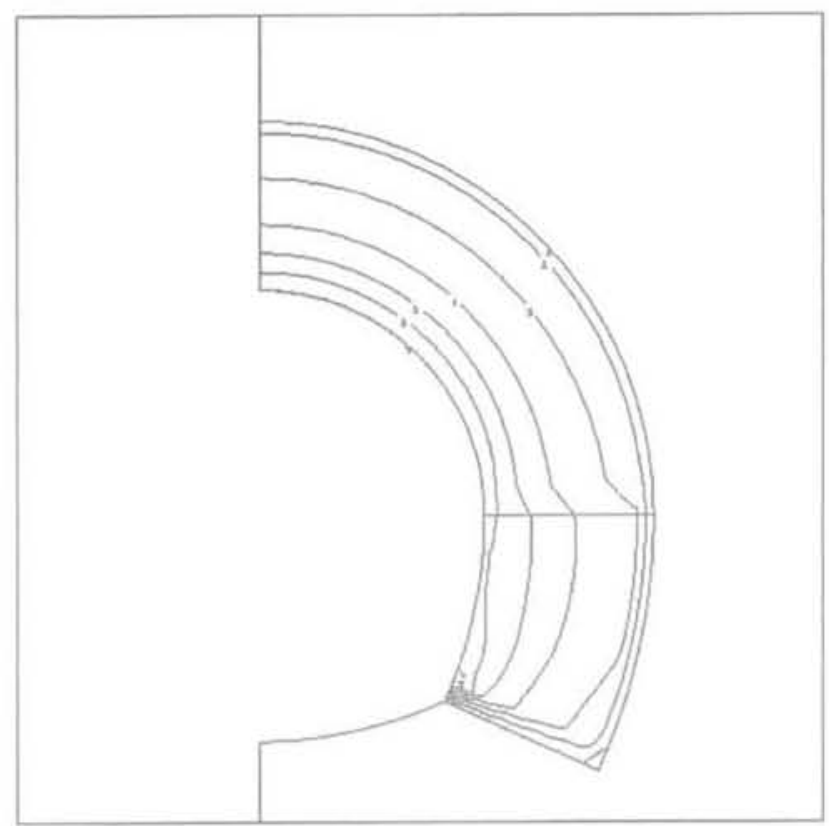

FG. 14 Isovaleurs de la contrainte $\underline{\sigma}^{r}$ dans la phase de renforcement.

Stress isovalues in the reinforced phase.

sur la paroi. Ceci est dû au choix que l'on a fait de retenir le modèle simplifié à adhérence parfaite entre les phases, qui permet de respecter les conditions aux limites en moyenne (c'est-à-dire sur les contraintes totales), mais ne permet pas d'imposer des conditions aux limites sur les contraintes partielles. La prise en compte de cinématiques différentes pour chaque phase et d'une loi d'interface entre elles, fait l'objet de la prochaine étape de l'intégration du modèle multiphasique dans CESARLCPC, et permettra de s'affranchir de ce type de problème.

La figure 15 représente les zones d'isovaleurs de la norme des déplacements; montrant logiquement que les mouvements les plus importants se situent à proximité de la galerie.

Nous avons reporté enfin, sur la figure 16, l'évolution du déplacement vertical du point situé à l'extrémité supérieure de la voûte en fonction du facteur multiplicatif $(1-\lambda)$ appliqué au chargement, pour l'ouvrage

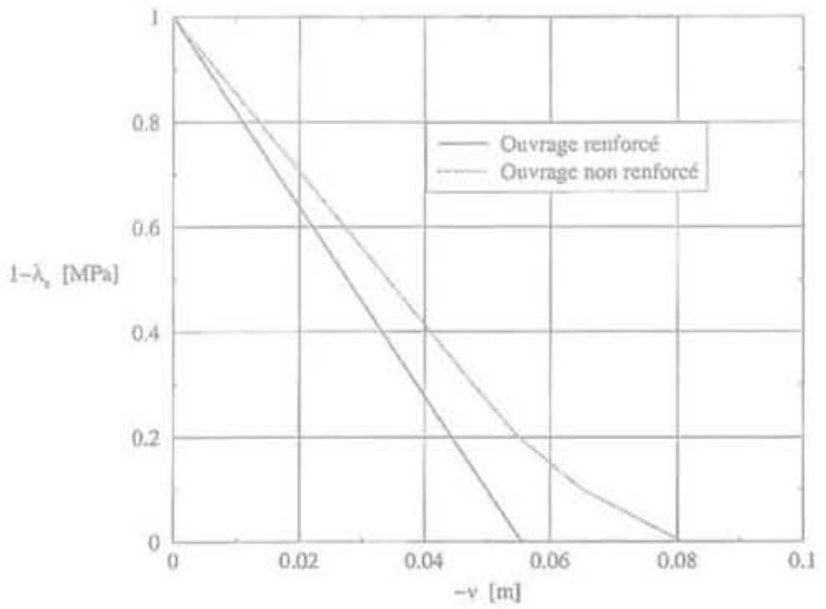

FG. 16 Convergence du tunnel en fonction du taux de déconfinement.

Tunnel convergence versus loading.

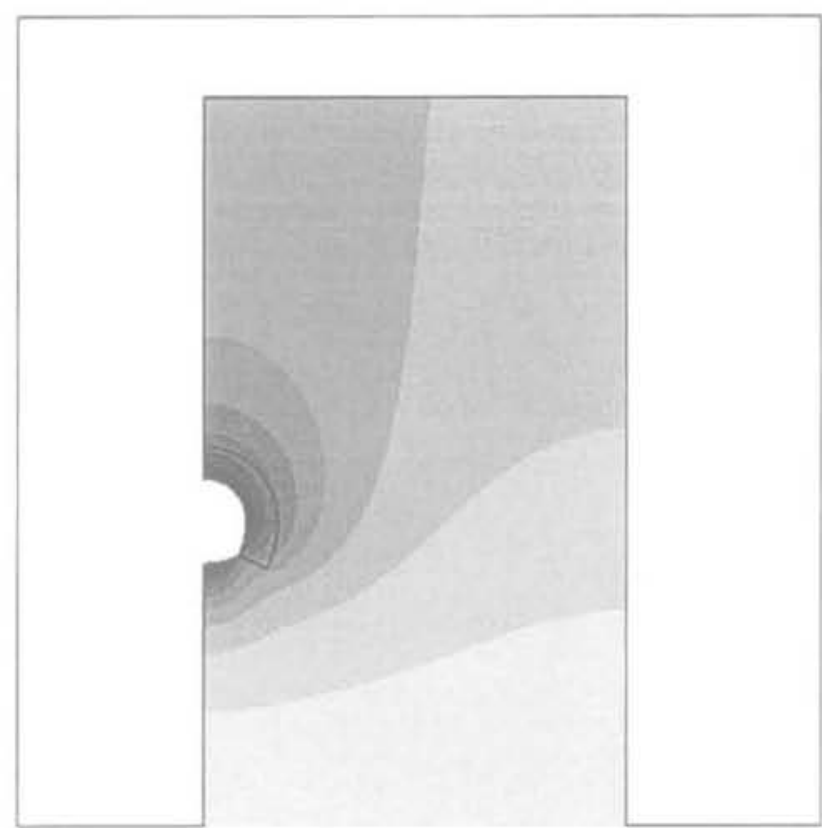

FG.15 Isovaleurs des déplacements totaux. Displacement isovalues.

renforcé et pour l'ouvrage non renforcé. On observe une convergence plus importante vers l'axe du tunnel, lorsque ce dernier n'est pas renforcé: les boulons réduisent le déplacement de ce point de plus de $30 \%$.

Une fois la construction du maillage effectuée, le temps de calcul est de quelques secondes. Cette souplesse d'utilisation permet d'utiliser le code, non seulement pour la vérification d'ouvrages, mais aussi au stade du dimensionnement.

\section{4 \\ Conclusion}

Ce travail a été l'occasion d'intégrer la méthode proposée par Sudret et de Buhan (1999) pour l'étude de sols renforcés dans le progiciel CESAR-LCPC. La version du code obtenue a permis d'étudier le comportement élastoplastique de plusieurs ouvrages renforcés typiques du domaine de la géotechnique, en deux et trois dimensions, et de montrer l'efficacité et la pertinence d'une telle approche.

L'état actuel du code ne permet pas de prendre en compte des cinématiques différentes pour chaque phase constituant l'ouvrage, mais cette version restreinte permet déjà, contrairement aux méthodes d'homogénéisation classiques, d'obtenir un matériau multíphasique écrouissable, bien que l'on considère au départ un comportement élastique parfaitement plastique pour les phases constituant ce matériau. En outre, il est important de noter que, en gérant numériquement séparément les phases, cette méthode est beaucoup plus rapide à mettre en œuvre, n'ayant pas à construire le comportement du matériau homogène équivalent, comme dans le cas d'une approche d'homogénéisation classique.

Cependant, lorsque l'on s'approche de la rupture, et de manière plus générale, lorsque le glissement du sol par rapport aux inclusions devient significatif (ce qui peut se produire si le contraste entre les proprié- 
tés du sol et des inclusions est trop grand), l'hypothèse d'adhérence entre les deux milieux continus qui représentent le sol renforcé devient plus discutable et les résultats donnés par le modèle s'écartent des résultats de mesures. Il est donc nécessaire de compléter le modèle de comportement utilisé, afin d'améliorer la prise en compte du comportement de l'interface entre les inclusions et le sol. C'est ce qui sera réalisé dans la deuxième phase de ce projet. En outre, la version actuellement programmée ne tient compte que des efforts de traction/compression dans les renforts. La possibilité de prendre en compte leur flexion (de Buhan et Sudret, 2000) devra donc être envisagée.

\section{$\overline{\text { Bibliographie }}$}

Bourgeois E., Garnier D., Semblat J.F., Sudret B., Al Hallak R. - $\alpha$ Approche par homogénéisation du comportement d'un front de taille de tunnel renforcé par boulonnage: comparaison avec des essais en centrifugeuse n. Revue française de génie civil, vol. $5, n^{*} 1,2001$, p. 9 38.

Buhan (de) P. - Approche fondamentale du calcul à la rupture des ouvrages en sols renforcés. Thèse d'État, Université de Paris 6, 1986.

Buhan (de) P., Sudret B. - $\alpha$ Micropolar Multiphase Model for Materials Reinforced by Linear Inclusions 3. European Journal of Mechanics, vol. 19, n4, 2000, p. 669-687.

Chow Y.K. - « Analysis of vertically loaded pile groups $\mathrm{x}$. International Journal of Numerical and Analytical Methods in Geotechnic, 17, 1986, p. 59-72.

Clancy P., Randolph M.F, « An approximate analysis procedure for piled raft foundations x. International Journal of Numeri- cal and Analytical Methods in Geotechnic, 10 (1), 1993, p. 59-72.

Coussy 0 . Mécanique des milieux poreux. Éditions Technip, 1991

Giroud J.-P, - «Settlement of a linearly loaded rectangular area y. Journal of Soi Mech. Found. Div. ASCE, 94 (SM4). 1968 , p. 813-832.

Greuell E. - Etude du soutènement pat boulons passif's dans les sols et les roches tendres par une méthode d'homogénéisation. Thèse de doctorat de l'École Polytechnique, Paris, 1993.

Greuell E.. Buhan (de) P., Panet M.. Salençon J. - "Comporternent des tunnels renforcés par boulons passifs in. XIIIth International Conference on Soil Mechanics and Foundation Engineering. New Delhi. Inde, 1993

Griffiths D.V., Clancy P., Randolph M.F. "Piled raft foundation analysis by finite elements \%. Proceedings of Computer methods and advances in Geomechanics (Beer, Booker et Carter, eds), Balkema, Rotterdam, 1991, p. 1153-1157.
Kraft L.M., Ray R.P., Kagawa T. - «Theoretical ( $\mathrm{t}$-z) curves n. J. Geo. Eng. Div. ASCE. 107 (GT11), 1981, p. 1543-1561.

Randolph M.F., Wroth C.P. "Analysis of the deformation of vertically loades piles o. J. Geo. Eng. Div.. ASCE, 104 (GT12), 1978, p. 1465-1488.

Randolph M.F. $-\alpha$ Design of piled raft foundation. Proceedings of Recent developments in laboratory and fieid tests and analysis of geotechnical problem ». Bangkik, vol. 5, 1983, p. 525-537.

Sudret B., Maghous S., Buhan (de) P., Bernaud D. $-\alpha$ Elastoplastic analysis of inclusion reinforced structure 1 . Metals and Materials, vol. $4, n^{\circ} 3,1998$, p. 252 255.

Sudret B. - Modélisation multiphasique des ouvrages renforcés par inclusions. Thèse de doctorat de l'École nationale des ponts et chaussées. Paris, 1999

Sudret B., Buhan (de) P. - « Modélisation multiphasique des matériaux renforcés par inclusions linéaires v. C.R. Acad. Sci. Paris, t. 327, série Ilb, 1999, p.7-12. 